\title{
Golgi GRASPs: moonlighting membrane tethers
}

\author{
This article was published in the following Dove Press journal: \\ Cell Health and Cytoskeleton \\ 3 May 2012 \\ Number of times this article has been viewed
}

\author{
Timothy Jarvela \\ Adam D Linstedt \\ Department of Biological Sciences, \\ Carnegie Mellon University, \\ Pittsburgh, PA, USA
}

Correspondence: Adam D Linstedt Department of Biological Sciences, Carnegie Mellon University, 4400 5th Avenue, Pittsburgh, PA 15213, USA

$\mathrm{Tel}+|4| 2268 \mid 249$

Fax + I 4I2 268 7I29

Email linstedt@cmu.edu
Abstract: The identification of mammalian Golgi reassembly stacking proteins (GRASPs) 15 years ago was followed by experiments implicating them in diverse functions, including two differing structural roles in Golgi biogenesis and at least two distinct roles in the secretion of proteins. GRASP55 and GRASP65 are localized to cis and medial/trans Golgi cisternae, respectively. They are both required for stacking of Golgi membranes in a Golgi reassembly assay. Depletion of either GRASP from cultured cells prevents the linking of Golgi membranes into their normal ribbon-like network. While GRASPs are not required for transport of secretory cargo per se, they are required for ER-to-Golgi transport of certain specific cargo, such as those containing a C-terminal valine motif. Surprisingly, GRASPs also promote secretion of cargo by the so-called unconventional secretory pathway, which bypasses the Golgi apparatus where the GRASPs reside. Furthermore, regulation of GRASP activity is now recognized for its connections to cell cycle control, development, and disease. Underlying these diverse activities is the structurally conserved N-terminal GRASP domain whose crystal structure was recently determined. It consists of a tandem array of atypical PSD95-DlgA-Zo-1 (PDZ) domains, which are well-known protein-protein interaction motifs. The GRASP PDZ domains are used to localize the proteins to the Golgi as well as GRASP-mediated membrane tethering and cargo interactions. These activities are regulated, in part, by phosphorylation of the large unstructured C-terminal domain.

Keywords: GRASP, review, membrane, tether, PDZ domain, secretory chaperone, unconventional secretion

\section{Introduction}

Proteins translated in association with the endoplasmic reticulum (ER) are packaged into vesicles for export to the Golgi apparatus. This protein cargo may bear exit signals promoting selective concentration during exit. ${ }^{1}$

In mammalian cells, ER exit sites are both centrally located and distributed throughout the cell periphery. Vesicles leaving the ER coalesce into the ER-Golgi intermediate compartment (ERGIC). ${ }^{2}$ From the ERGIC, there is both recycling to the ER and transfer of cargo-bearing membrane to the centrally located Golgi apparatus. ${ }^{3}$

The Golgi apparatus is structured to promote sequential processing of the arriving cargo. ${ }^{4}$ Flattened membranes called cisternae are in close apposition to one another, forming a stack. The first Golgi enzymes to act on the cargo are enriched in the entry, or cis-cisternae. Enzymes enriched in the medial cisternae of the stack carry out the next processing steps. The last events occur in the trans-cisternae and trans-Golgi network (TGN). In the TGN, the processed cargo is packaged for exit from the Golgi apparatus. 
The stacked structure of the Golgi apparatus is evident in most eukaryotes.

Mammalian Golgi stacks are positioned near the centrosome-based microtubule-organizing center and they are laterally linked to form a ribbon-like membrane network. ${ }^{5}$ Dynamic tubular membranes connect adjacent analogous cisternae within this network promoting uniform, yet cisternae-specific distribution of Golgi enzymes across the entire Golgi ribbon. Discontinuities, if prolonged, lead to nonuniform enzyme distribution and under-processing of secretory cargo. ${ }^{6}$ Positioning of Golgi membranes near the centrosome allows the secretion to be efficiently directed to the most proximal plasma membrane domain or cell leading edge. ${ }^{7}$ This directed secretion is needed for cell polarity and cell migration for wound repair. It is also involved in muscle differentiation, immunological synapse formation, and neuritic process extension. ${ }^{8-10}$

In preparation for mitosis, the mammalian Golgi ribbon is unlinked, and the resulting stacks then vesiculate, leaving dispersed vesicles and clusters of vesicles. ${ }^{1-13}$ These vesicles and vesicle clusters are partitioned equally into daughter cells, whereupon they reassemble into the stacked and laterally linked interphase membrane network. The Golgi ribbon is also extensively fragmented during apoptosis, and unlinking of the ribbon is thought to occur transiently during reorientation of the organelle as cells define a new leading edge. ${ }^{14,15}$
Interestingly, certain secretory cargo molecules entirely bypass the Golgi apparatus en route to secretion. ${ }^{16,17}$ Examples include fibroblast growth factors 1 and $2,{ }^{18,19}$ interleukins $1 \alpha$ and $\beta,{ }^{20,21}$ integrin $\alpha$ PS $1,{ }^{22}$ acyl-CoA binding protein, ${ }^{23-26}$ and galectins ( 1 and 3$) .{ }^{27-30}$ Unconventional secretion occurs by multiple routes. For some unconventional cargo, secretion begins with transmembrane proteins translated in association with the ER or cytosolic proteins internalized into autophagic membranes. ${ }^{31}$ Membranes derived from the ER or the autophagic membranes then dock and fuse directly with the plasma membrane to transfer their content.

Since their discovery 15 years ago, experimental evidence indicates that mammalian Golgi reassembly stacking proteins (GRASPs) may participate in a surprising number of the processes just described (Figure 1). Mammalian cells express two GRASPs and both are encoded by distinct genes. GRASP65 is localized to cis-Golgi cisternae and GRASP55 is localized to medial and trans-Golgi cisternae. ${ }^{32}$ The GRASPs contain a conserved N-terminal GRASP domain. Simpler eukaryotes express a single GRASP, which is also defined by the presence of this domain. The key feature of the GRASP domain is its two PSD95-DlgA-Zo-1 (PDZ) domains, which interact with PDZ ligands in partner proteins (Figure $2 \mathrm{~A}$ and $\mathrm{B}$ ). Following the GRASP domain, GRASPs have a nonconserved, serine and proline rich (SPR) domain comprising about half of the protein (Figure 2A). A simplifying assumption is

Unconventional secretory pathway

Conventional secretory pathway

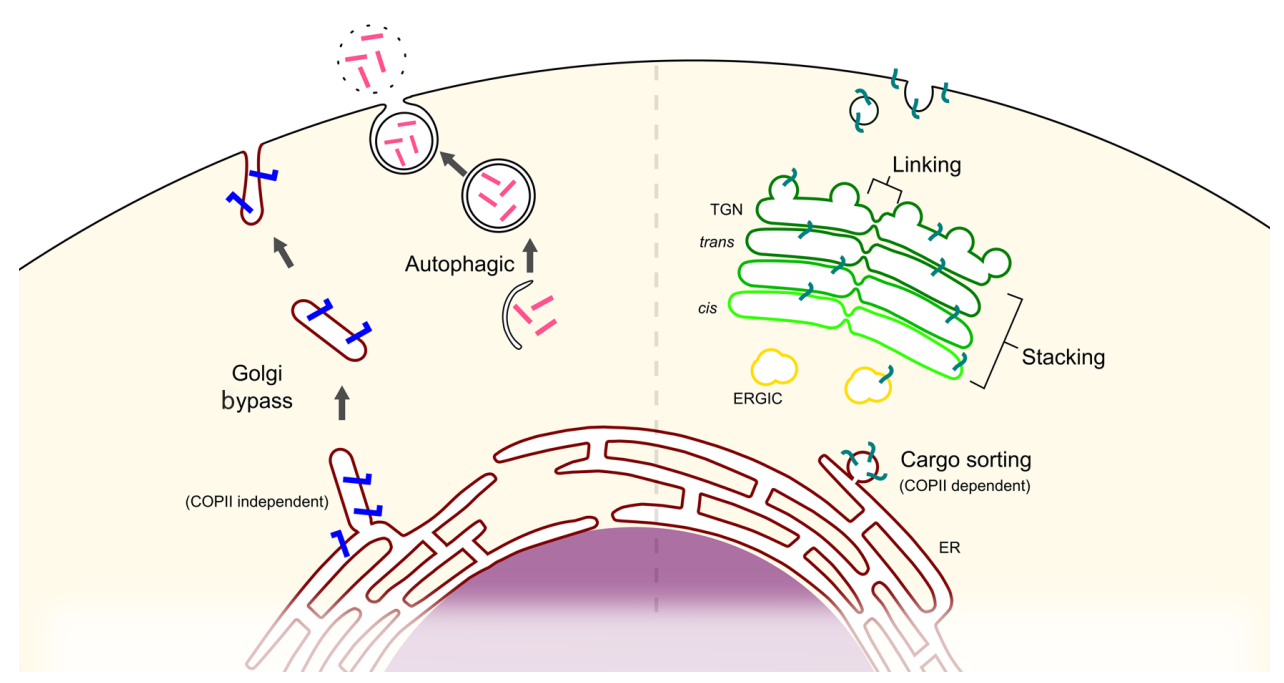

Figure I Overview of unconventional and conventional secretory pathways.

Notes: Three types of cargo are shown. Unconventional transmembrane cargo (blue) is shown exported from the ER in a COPII independent fashion followed by fusion of its carrier with the plasma membrane. Cytosolic cargo (pink) is first internalized in autophagic membranes. Traditional cargo (teal) undergoes COPII-mediated budding, transfer from the ER Golgi intermediate compartment to the Golgi, and export from the Golgi to the plasma membrane. Linking and stacking are, respectively, homotypic and heterotypic membrane interactions that contribute to Golgi ribbon architecture.

Abbreviations: ER, endoplasmic reticulum; ERGIC, ER-Golgi intermediate compartment; TGN, trans-Golgi network. 


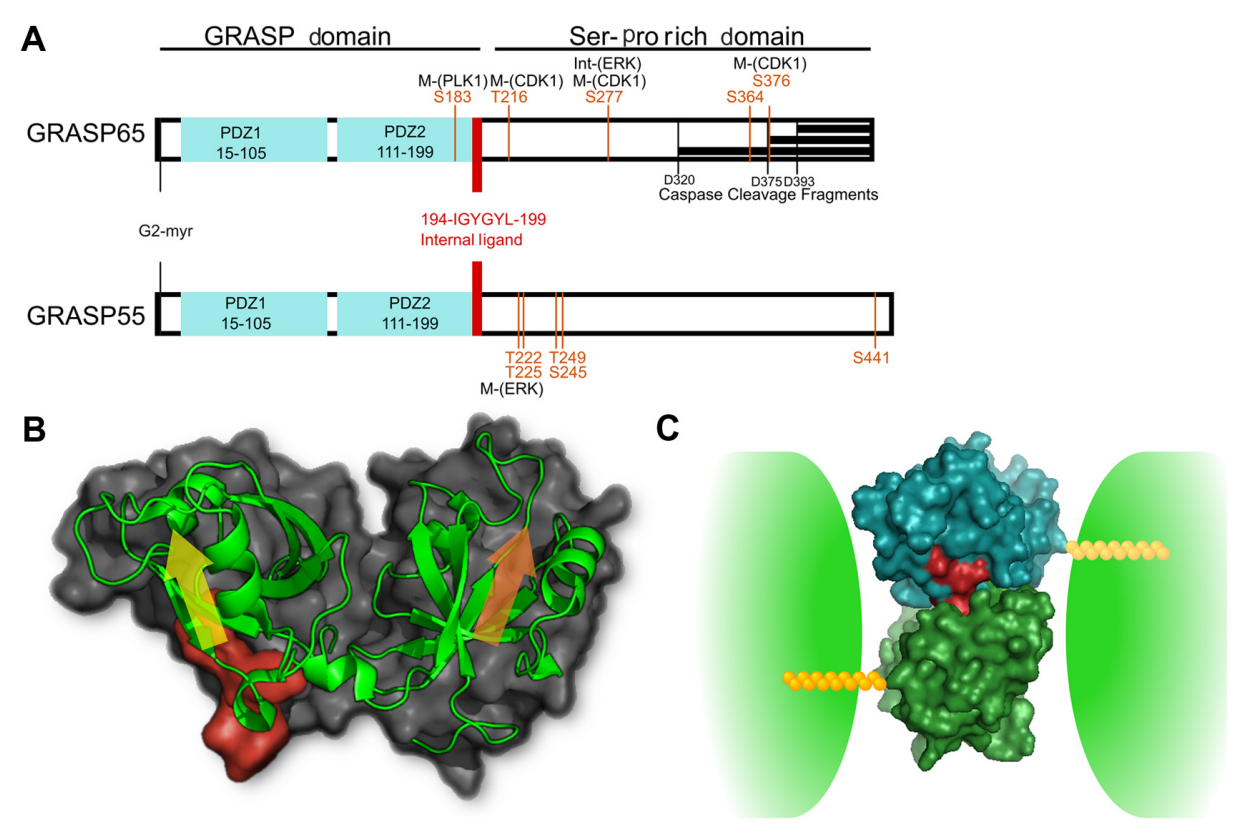

Figure 2 Mammalian GRASP65 and GRASP55. (A) Schematic of mammalian GRASP functional domains and features. N-terminal myristoylation (on glycine at position 2), PDZI and PDZ2 (blue), phosphorylation sites (orange), internal ligand (red), and caspase cleavage sites (black) are shown with indicated residue numbers. The implicated kinases active at interphase or mitosis are also indicated. (B) A cartoon representation of the GRASP domain of GRASP55 I-208 overlaid on space fill model. PDZI (orange arrow) and PDZ2 (yellow arrow) binding grooves are highlighted. The internal ligand is highlighted in red. (C) Schematic of GRASP domain tethering mechanism. Two apposed Golgi cisternal rims are shown (green) each bearing a GRASP protein. The internal ligand (red) of one GRASP domain is docked in the PDZI binding pocket of the second GRASP domain. Myristoylation (yellow) anchors the domains and is hypothetically placed.

Abbreviation: GRASP, Golgi reassembly stacking protein.

that the GRASP domain mediates functional interactions regulated by the SPR domain.

\section{GRASP structural features The GRASP domain}

The GRASP domain is a striking feature conserved across GRASP homologues. It is comprised mostly of two PDZ domains in tandem (Figure 2B). PDZ domains share a conserved globular structure in which an $\alpha$-helix and $\beta$-strand form a hydrophobic binding groove. Many proteins contain PDZ domains, and most PDZ-containing proteins contain multiple such domains that are present in arrays. The domain mediates protein-protein interactions and, as such, its presence in multiple copies confers the ability to scaffold the assembly of large complexes. ${ }^{33}$ Typically, the ligands that bind the groove fall into one of three categories. Canonical ligands are present at a protein's C-terminus. The four $\mathrm{C}$-terminal residues insert into the PDZ groove, forming a $\beta$-strand that completes a $\beta$-sheet. The carboxyl group at the $\mathrm{C}$-terminus is coordinated within the PDZ binding pocket by a Gly-Leu-Gly-Phe sequence and water molecule. ${ }^{34}$ The second and third types are internal sequences. They may form a $\beta$-finger with a tight hairpin and usually an acidic amino acid to mimic the free C-terminus ${ }^{35}$ or they might simply be flexible sequence stretches that contain glycines, for example, that decrease steric constraints and project side chains into the pocket. ${ }^{36,37}$

The structure of the GRASP55 GRASP domain was recently solved ${ }^{38}$ and unexpectedly revealed that the GRASP PDZ domains lack the typical $\beta \beta \beta \alpha \beta \beta \alpha \beta$ secondary structure organization of eukaryotic PDZ domains. Instead, the two PDZ domains are circularly permuted, which has two important consequences. First, although not evident among metazoan PDZ domains, circular permutation is present in prokaryotic PDZs. In fact, the overall structures of the GRASP PDZ domains align remarkably well with prokaryotic PDZ domains as well as with one another. ${ }^{18}$ Second, this unusual arrangement of a metazoan PDZ revealed that the key $\beta 2$ strands of the binding grooves lay outside the previously predicted PDZ-like regions. This calls into question the interpretation of experiments based on incorrect assignment of the domains. In fact, a recent study failed to include the key $\beta 2$ strands in its GRASP PDZ domain constructs even though this work was published after the structure was known. ${ }^{39}$ Inexplicably, the constructs were binding competent, but this binding could not have been due to a PDZ interaction.

Although the two PDZ domains in the GRASP domain are similar in overall structure, there are significant differences in the binding grooves, which reflect the specificities of their interactions. The interface between the second $\alpha$-helix 
and second $\beta$-strand of the PDZ1 binding domain contains a deep depression resembling a pocket. ${ }^{38}$ In contrast, the PDZ2 groove contains a phenylalanine occluding the pocket. Evidence suggests that while PDZ2 of GRASP65 binds a C-terminal ligand present in the coiled-coil Golgi protein GM130, PDZ1 interacts with a novel type of internal PDZ ligand. Remarkably, this ligand is present on the surface of the PDZ2 domain and forms a conspicuous surface protrusion that appears to fit into the pocket of PDZ1 (Figure 2C). As described in more detail below, the separation of function of the two PDZ domains in GRASP65 allows targeting of the protein to the Golgi via PDZ2 and homodimer formation via PDZ1. Together these activities allow the protein to mediate homotypic membrane tethering. Residues flanking PDZ2 may also stabilize the interaction of PDZ2 with GM130. Mutation of these residues blocks binding to GM130, ${ }^{40}$ and flanking regions are known to stabilize the ligand-pocket interaction of other PDZ domains by making the PDZ domain less dynamic. ${ }^{33}$ Paralleling the activities of GRASP65, GRASP55 self-interacts and binds a coiledcoil Golgi protein, golgin45. Nevertheless, it remains to be determined whether these activities map to PDZ1 and PDZ2, respectively, and whether the golgin45 interaction mediates Golgi localization of GRASP55. ${ }^{41}$

\section{$\mathrm{N}$-terminal membrane attachment}

The mammalian GRASPs are myristoylated at their N-termini. This modification is required along with golgin binding for their localization to the Golgi. ${ }^{42}$ Thus, dual contact with the membrane stabilizes membrane binding. The N-terminal myristic acid is immediately adjacent to the PDZ1 module. Mutation of the glycine residue that becomes myristoylated blocks membrane tethering by PDZ1, even if the protein is stably anchored to the membrane by other means. Substituting a transmembrane domain for the myristoylation site restores activity, which indicates that the N-terminus of PDZ1 must be membrane anchored for PDZ1-mediated membrane tethering. ${ }^{43}$ Interestingly, membrane binding of the N-terminus is conserved, even though certain species express GRASPs without the myristoylation sequence. ${ }^{44,45}$ The GRASP homologue in Plasmodium falciparum expresses a splice variant with an N-terminal signal anchor, and the Saccharomyces cerevisiae version has an amphipathic helix that is acetylated to mediate membrane association.

There appears to be additional significance to the dualanchoring of GRASP65 by the N-terminal myristic acid and GM130 binding. ${ }^{6}$ The two contact points of membrane binding might orient the GRASP65 homotypic binding interface to promote trans interactions across two membranes. ${ }^{46}$ Subsequent membrane fusion then imparts a torque on the complex because of the dual anchors. In other words, membrane rearrangement forces an unfavorable cis configuration leading to partner dissociation. Because they are relatively weak, PDZ interactions ${ }^{47}$ might lend themselves to regulation by membrane dynamics. Interestingly, by their shared requirement for dual anchoring, localization of the tether and tether activity are linked. Thus, only dually anchored GRASP65 molecules are on the Golgi, thereby ensuring that cis interactions do not interfere with trans pairing.

GRASP55 has at least one additional fatty acid modification. It is palmitoylated ${ }^{48}$ although the functional relevance of this modification is unclear. The longer acyl chain could affect the distribution and preference of GRASP55 in the membrane, making it prefer the thicker, late Golgi membranes. ${ }^{49}$

\section{Internal ligand}

GRASP55 and GRASP65 share a conserved sequence stretch IGYGYL at the end of PDZ2 that binds PDZ1 ${ }^{38,50}$ (Figure $2 \mathrm{~A}-\mathrm{C}$ ). The glycines likely contribute to rotational flexibility, allowing the surface projecting tyrosine and leucine side chains to fit precisely into the deep pocket present in the PDZ1-binding groove. ${ }^{38}$ Extensive van der Waals contacts may provide the stability to the interaction, nullifying the need for coordination of a carboxyl group seen in other PDZ interactions. Because the proteins do not bind one another, it is puzzling that the surface projecting ligand is identical in GRASP65 and GRASP55. There may be residues flanking the ligand sequence that play an important role in ligand specificity. Importantly, the GRASP ligand is a target of mitotic regulation. In GRASP65 a nearby serine is phosphorylated during mitosis likely inducing a conformational change that blocks the ligand from binding PDZ1. ${ }^{50}$

\section{Serine proline-rich (SPR) domain}

The C-terminal half of the GRASP proteins is enriched in serine and proline residues. This region appears to be unstructured. In striking contrast to the GRASP domain, the SPR domain is not conserved at the sequence level. Noteworthy in this region are multiple phosphorylation and caspase cleavage sites. ${ }^{11,14,51-56}$ Cyclin-dependent kinase 1/cyclin B (CDK1), a MEK/ERK cascade, and Polo-like kinase 1 (PLK1) each phosphorylates one or both GRASP proteins. ${ }^{51-53}$ ERK directly phosphorylates GRASP55, and inhibition of its upstream activator MEK1 blocks both GRASP55 phosphorylation and G2-phase Golgi unlinking. ${ }^{11,52,53}$ Furthermore, mutation of ERK phosphorylation sites in GRASP55 to mimic the phosphorylated 
state blocks GRASP55 activity in both Golgi ribbon formation and self-association. ${ }^{52}$ GRASP65 is directly phosphorylated at multiple sites by CDK1, ERK, and PLK1, and phosphorylation blocks its homo-oligomerization in vitro. ${ }^{53,57-59}$ Interestingly, the PLK family of kinases initially binds substrates and becomes activated through their Polo box domains. Once activated, the kinases can phosphorylate distant sites. ${ }^{60-62}$ In the case of GRASP65, mitotic phosphorylation of the SPR domain creates a PLK1 binding site. ${ }^{63}$ The activated PLK1 then phosphorylates a site adjacent to the internal GRASP65 PDZ ligand and blocks its ability to mediate GRASP65 self-interaction. ${ }^{50}$

\section{Structuring the Golgi The role of GRASPs in Golgi structure}

Stacking of Golgi cisternae is heterotypic in that Golgi stacks consist of cisternae of differing membrane compositions. Stacking connections might be initiated as new cis-cisternae form and might be broken as the membranes of trans-cisternae remodel, giving rise to budding membranes. During mammalian cell mitosis, as the Golgi vesiculates, the stacked architecture of the Golgi is lost, which suggests inhibition of the stacking mechanism. In contrast to stacking, Golgi ribbon formation is a homotypic reaction. Analogous cisternae among Golgi stacks are dynamically linked to one another by tubular membrane projections that undergo fusion and fission. Specificity of fusion in ribbon formation is needed to preserve compositional differences between cis-, medial-, and trans-cisternae. The lateral ribbon-forming contacts between analogous cisternae are disrupted as cells prepare for cell division and rebuilt after cell division. ${ }^{64}$

Originally, GRASP65 and then GRASP55 were shown to be required individually for the in vitro assembly of stacked Golgi membranes. ${ }^{42}$ Surprisingly, however, knockdown of either GRASP65 or GRASP55 in cultured cells leaves the Golgi with normal stacks. ${ }^{6,11}$ Only depletion of both proteins perturbs the stacked architecture ${ }^{65}$ GRASP proteins have the ability to link membranes through their homotypic interactions, but the membrane junctions evident when GRASP proteins are used to tether mitochondrial membranes to one another are morphologically distinct from those of Golgi cisternae. ${ }^{43}$ Even more perplexing, Saccharomyces cerevisiae does not have a stacked Golgi and yet expresses a GRASP homologue, and Pichia pastoris has a stacked Golgi, yet its stacked architecture does not depend on GRASPs. ${ }^{66-68}$ In Drosophila, depletion of the lone GRASP homologue dGRASP decreases but does not abolish cisternal stacking. ${ }^{69}$ Overall, it seems that another mechanism is primarily responsible for Golgi stacking, the GRASP proteins providing an additional contribution.
Mammals and other vertebrates that form Golgi ribbon networks express two GRASP proteins. Depletion of either breaks the ribbon into individual Golgi stacks. Because each protein interacts only with itself, the proteins could act in parallel reactions: GRASP65 supports membrane fusion to link laterally and elongate cis-cisternae, and GRASP55 maintains these contacts in medial cisternae. From an evolutionary perspective, functional divergence that gives rise to compartment specific tethering by the GRASP proteins might be for the maintenance of Golgi subcompartments in the face of microtubule-based motility, thereby bringing Golgi ministacks into close proximity in the region of the microtubule organizing center. In lower eukaryotes, a single GRASP gene is present and Golgi membranes, even when present as stacked cisternae, are neither confined to a central position nor fused laterally to form a ribbon-like membrane network. Possibly, ribbon formation in vertebrates is a more extreme form of cisternal elongation carried out by simpler eukaryotes. ${ }^{70}$ If so, homotypic membrane-tethering mediated by membraneanchored PDZ1 could represent the fundamental mechanism of GRASP65 action. The physical distance separating Golgi elements in simpler eukaryotes may prevent lateral fusion which, given the presence of only a single GRASP, might otherwise impair maintenance of subcompartment identity.

\section{GRASP-tethering mechanism}

GRASPs contribute to Golgi structure by homotypic oligomerization, an ability that was first observed for purified GRASP65 either in isolation or on the surface of beads. ${ }^{57,58}$ The GRASP domain mediates oligomerization. In order to understand the mechanism of GRASP-mediated tethering on the surface of membranes and in physiological conditions, GRASP65 has been targeted to the outer membrane of mitochondria. ${ }^{43}$ Paralleling its activity at the Golgi, GM130 recruits GRASP65, and GRASP65 is necessary and sufficient for mitochondrial tethering, which depends on the PDZ1binding groove and the PDZ2 surface-projecting ligand. The same mutations of these elements that block mitochondrial clustering also block GRASP-mediated Golgi ribbon formation. Thus, the mechanism involves an internal PDZ ligand within a GRASP65 partner on one membrane binding to the first of two PDZ binding pockets in a GRASP65 partner on the opposing membrane (Figure 3A). The binding groove of PDZ2 is expendable for tethering membranes, but it is needed to localize GRASP65 to cis-cisternae by binding GM130. Both the N- and C-termini of the GRASP domain require anchoring to the membrane for efficient tethering, the former by myristoylation and the latter by a golgin. 
This orients the internal ligand and PDZ1 pocket into a conformation that promotes a trans-interaction between GRASPs on separate membranes. ${ }^{46}$ Loss of either anchor point allows free rotation of the GRASP molecule in the plane of the membrane, which allows interactions in cis and decreases the propensity for functional trans-pairing.

As mentioned above, the crystal structure of the GRASP domain yields a model for the tethering interface. The internal ligand present on the surface of PDZ2 projects its side chains into the deep binding pocket of PDZ1. ${ }^{38} \mathrm{~A}$ significant feature of the GRASP domain structure is that the internal ligand for self-interaction is on the surface opposite that of the PDZ1 groove to which it binds. This provides a structural explanation for the observed tendency of GRASP65 to form multimers ${ }^{58}$ and suggests that interdigitation of the molecules may occur during tethering.

\section{Regulation of GRASP activity Mitotic regulation of GRASP tethering activity}

The homotypic tethering activity of both GRASP65 and GRASP55 is regulated during the cell cycle. As mentioned, both GRASP65 and GRASP55 are phosphorylated during mitosis.
GRASP65 is a major target of Plk-1. ${ }^{42}$ GRASP55 is phosphorylated by MEK/ERK. ${ }^{11,52,53}$ In the case of GRASP65, a twostep phosphorylation process inhibits its homotypic tethering activity (Figure 3B). First, the SPR region is phosphorylated by ERK (in late G2) or CDK1 (in M phase), resulting in a docking site for Plk-1. ${ }^{15,63}$ Plk-1 then phosphorylates Ser189, which has been shown to block homotypic tethering and cause unlinking of the Golgi ribbon. ${ }^{50}$ The proximity of Ser-189 to the internal ligand suggests that phosphorylation of Ser-189 causes a conformational change in the ligand that prevents its binding to the PDZ1 pocket. GRASP55 also has a serine at 189 but whether it is mitotically phosphorylated at this position is not known. GRASP55 does not appear to interact with Plk-1 during mitosis. ${ }^{63}$

\section{GRASP regulation during cell motility}

Certain cell types respond to an external motility cue by repositioning their microtubule-nucleating centrosome. Golgi membranes also move, presumably because cytoplasmic dynein keeps them near the minus ends of microtubules. Intriguingly, expression of a version of GRASP65 that cannot be phosphorylated by ERK blocks cellular reorientation to a scratch wound. ${ }^{15}$ Because GRASP65 maintains

A

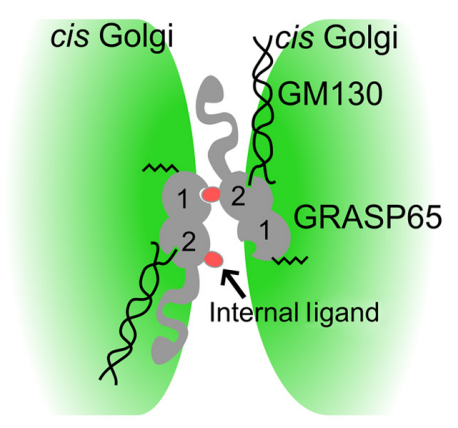

B
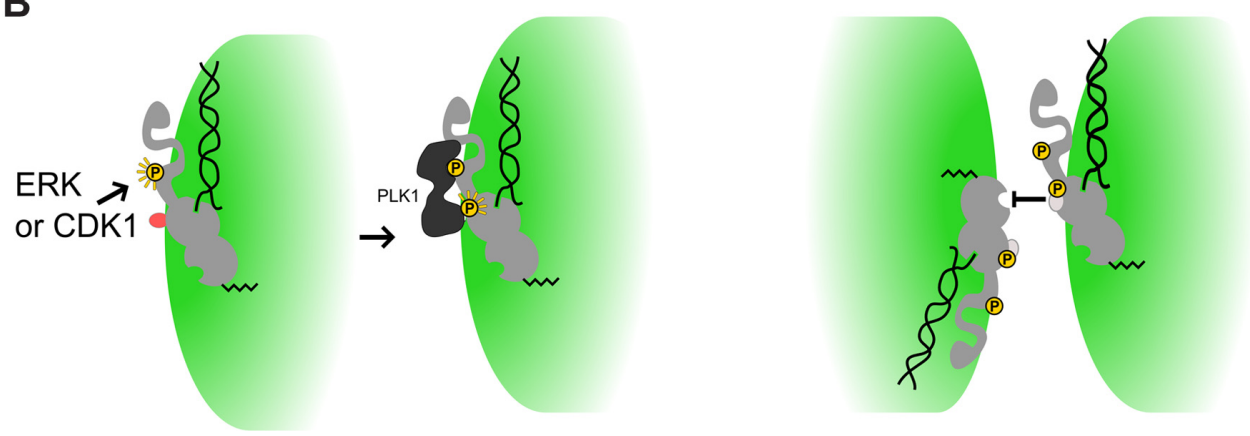

Figure 3 GRASP65 tethering and 2 step phosphoregulation. (A) Schematic diagram of GRASP65 tethering at the rims of two cis-Golgi cisternae (green). The internal ligand (shown as a red protrusion) of one GRASP65 binds to the PDZI pocket of the other. Each is anchored to the membrane through N-terminal myristoylation and a PDZ2GMI 30 interaction. (B) Two step model for GRASP65 inactivation. ERKI/2 or CDKI phosphorylates a site in the SPR domain creating a binding site for PLKI. PLKI (shown in dark grey) docks and phosphorylates Ser-189 near the internal ligand. This phosphorylation causes a conformational change in the ligand, which prevents its binding to the PDZI pocket.

Abbreviation: GRASP, Golgi reassembly stacking protein. 
Golgi structure and is phospho-inhibited, it is thought that GRASP65 is phosphorylated under these conditions to break transiently the Golgi membrane network, thereby removing a physical barrier that Golgi membranes might exert over centrosomal movement. This view is supported by the finding that Golgi disassembly by brefeldin A treatment bypasses the requirement for GRASP65 phosphorylation. ${ }^{15}$

\section{GRASP regulation during development}

In migrating granule neurons of the developing nervous system, polyubiquitination of GRASP65 PDZ1 by the Golgi-localized Cul7-Fbxw8 E3-ubiquitin ligase decreases GRASP65 protein levels. $^{71}$ Interestingly, reducing GRASP65 levels causes an increase in dendritic size and branching. Because GRASP65 knockdown increases Golgi fragmentation, possibly increasing its secretory ability, ${ }^{72}$ it may be that ubiquitination of GRASP65 is a regulated step that governs morphogenesis of the Golgi apparatus and development of dendrites in the brain.

\section{GRASPs and AIDS progression}

Hiyoshi and colleagues have shown that GRASP65 is a critical downstream target in human immunodeficiency virus (HIV) to acquired immunodeficiency syndrome (AIDS) progression in macrophages. HIV-1 Nef is a steric activator of Hck, a Src kinase in macrophages. By reducing the surface level of the receptor Fms, Hck inhibits macrophage colony stimulating factor signaling. ${ }^{73}$ The reduced signaling disables the anti-inflammatory state, ${ }^{74}$ thereby allowing more viral replication. ${ }^{75}$ The reduction in Fms surface levels is attributed to Golgi accumulation of a hypo-N-glycosylated form of the protein. ${ }^{48}$ The cause of this accumulation appears to be disruption of the Golgi ribbon through phosphorylation of GRASP65 by ERK, ${ }^{76}$ which is consistent with other experiments showing reduced glycosylation upon perturbation of the Golgi ribbon. ${ }^{6}$ In sum, inhibition of GRASP65-mediated cisternal linking appears to play a role in the virulence of AIDS. These experiments also show that glycosylation defects arising from perturbed Golgi ribbon maintenance can have physiologically significant effects.

\section{Chaperoning secretion and processing \\ C-terminal valine motif cargoes}

Although GRASPs are not required for traffic of model cargo, such as the viral protein VSVG, the situation is different for processing and transport of specific proteins.
One type of protein that is dependent on GRASPs contains a C-terminal valine motif. These include CD8- $\alpha$, Frizzled $4,{ }^{77}$ and TGF- $\alpha .{ }^{348}$ Their efficient transport is thought to involve GRASP65 chaperoning ER-to-Golgi transit and GRASP55 chaperoning intra-Golgi trafficking (Figure 4).

The C-terminal valine motif matches the minimal consensus for a C-terminal PDZ-ligand, and these cargos bind PDZ1 of both GRASP65 and GRASP55. ${ }^{48,77}$ In the absence of GRASP65, the cargo molecules accumulate at ER exit sites, whereas GRASP55 depletion results in the retention of these cargoes at the Golgi. ${ }^{77}$ The interaction with GRASP65 might serve as an exit signal, as the yeast GRASP has been shown to localize to exit sites and interact with the inner COPII coat complex sec23/24. ${ }^{44,68}$ However, it is unknown how GRASP55 depletion decreases exit from the Golgi of C-terminal valine motif cargoes. ${ }^{77}$ One hypothesis is that interaction with GRASP55 is needed to free these cargoes from their interaction with GRASP65, which holds them in the cis-Golgi.

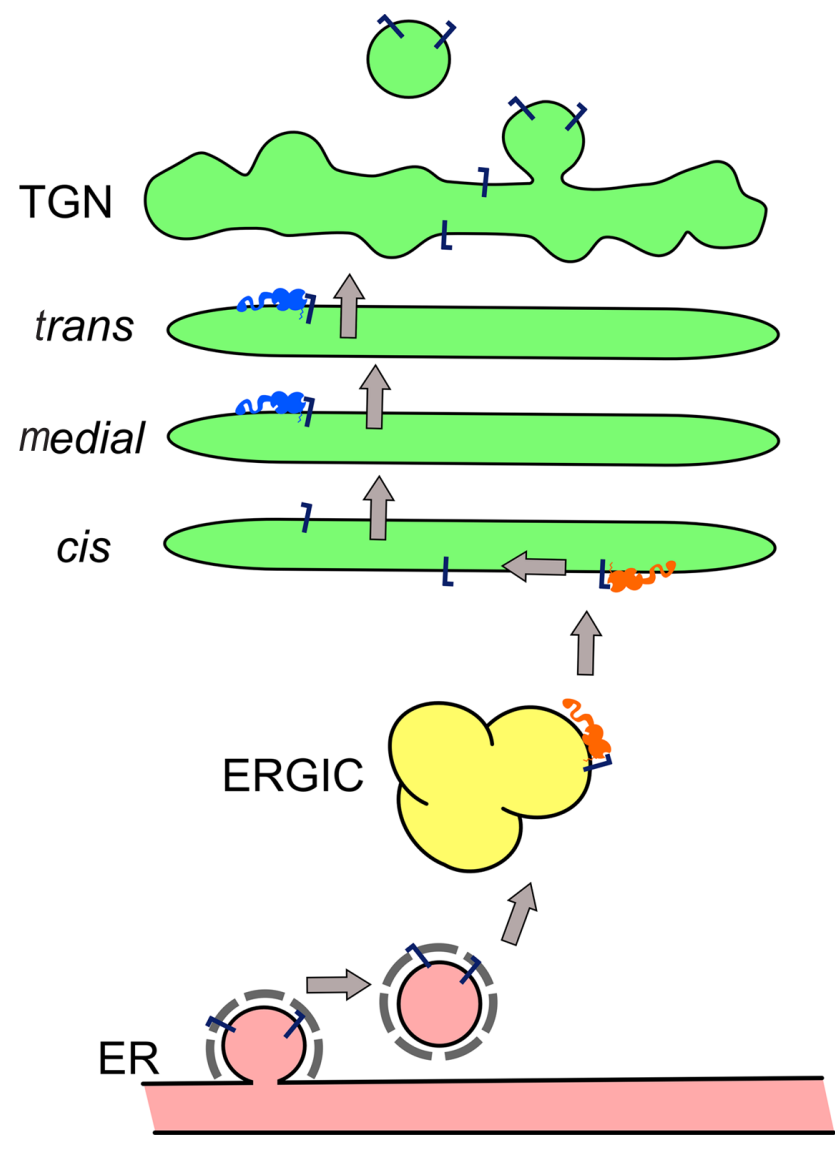

Figure 4 GRASP65 and GRASP55 work sequentially to promote C-terminal valine motif cargo through the secretory pathway.

Notes: Cargo with a C-terminal valine motif interacts with GRASP65 (orange) at the ERGIC to promote transport to the cis Golgi somehow. Once at the Golgi, GRASP55 (blue) binds the cargo and somehow promotes its transport through the stack.

Abbreviations: ERGIC, ER-Golgi intermediate compartment; GRASP, Golgi reassembly stacking protein;TGN, trans-Golgi network. 
An intriguing aspect of these findings is that cargo levels could influence Golgi ribbon integrity. ${ }^{78,79}$ Increasing occupancy of PDZ1 by C-terminal valine-bearing cargo would prevent homotypic GRASP interactions. Consequently, the ribbon would transiently fragment, thereby exposing greater surface areas for the purpose of cargo transit.

\section{Cargo adaptors}

GRASPs also interact with the p24 family of cargo receptors. ${ }^{80}$ This family of cargo receptors is important for secretion of lumenal cargo, and they interact with both the COPI and COPII vesicle coats. ${ }^{81}$ Interestingly, their cytoplasmic C-termini also match a minimal consensus for PDZ ligands, and they bind the GRASP proteins. Loss of GRASP binding by mutation of the C-terminal VV to AA results in an increased surface expression of these cargo receptors ${ }^{80}$ and would presumably decrease the secretion efficiency of p24-specific cargoes. GRASP65 was found in complexes containing both GM130 and p 24 family members. GRASP 55 was found in complexes with either golgin-45 or p24 family members, but not both at the same time.

\section{Processing efficiency}

Interactions between GRASPs and cargo can also increase cargo-processing efficiency. A minor population of GRASP55 is found in cells interacting with the cytoplasmic domain of furin and the cytoplasmic domain of a matrix metalloproteinase (MT1-MMP) ${ }^{82}$ The latter involves the sequence LLY near the C-terminus of MT1-MMP possibly binding PDZ2 of GRASP55. Furin also binds PDZ2, which suggests that dimerization of GRASP55 through PDZ1 generates a scaffold that brings furin and MT1-MMP together. This allows furin to cleave to the N-terminal inhibitory prodomain peptide of MT1-MMP. Because MMPs are often regulated during development, ${ }^{83}$ binding of GRASP55 to MT1-MMP could be developmentally regulated.

\section{Unconventional secretion}

While the majority of secreted proteins follow the canonical secretory pathway, some proteins follow an unconventional pathway. This pathway includes both cytoplasmic and membrane spanning cargo. The former lack a signal sequence and do not incorporate into the ER during synthesis, whereas the latter are translated in association with the ER. Transport of both types for secretion excludes the Golgi apparatus, and despite being predominately Golgi localized, GRASP proteins are required in this process (Figure 5). Unconventional secretion is regulated by stress. ${ }^{17}$

\section{Autophagic secretion}

In Dictyostelium discoideum, knockout of its GRASP homologue GrpA blocks secretion of acyl coenzyme A binding protein (AcbA) causing decreased sporulation. ${ }^{25} \mathrm{AcbA}$ lacks a signal sequence, which indicates that it is secreted unconventionally. AcbA secretion is induced by the spore differentiation factor 2 (SDF-2), and there is cytoplasmic build-up of AcbA in the absence of GrpA. Further analysis shows that stimulation with SDF-2 results in AcbA incorporation into vesicles prior to secretion and that GrpA is required for the fusion of these vesicles, not their formation. ${ }^{26}$ Significantly, the secretion of the AcbA homologue in yeast, Acb1, requires the yeast GRASP Grh1, as well as autophagosomal and early endosomal machinery. ${ }^{23,24}$ It also requires Bug1, the yeast homologue to GM130, which suggests that, in this pathway too, the GRASP requires a golgin for its attachment to membranes.

During the inflammatory response in macrophages, caspase- 1 is activated and cleaves the precursor to the interleukin cytokine IL-1 $\beta$. The cytokine is processed in the cytosol and secreted, bypassing the normal secretory pathway. Induction of autophagy stimulates secretion causing a partial redistribution of GRASP55 from the Golgi to the autophagic membranes. ${ }^{21}$ Knockdown of GRASP55 reduces the number of autophagic punctae and reduces secretion. The mechanism of GRASP55 in autophagy related unconventional secretion is currently unknown.

\section{Golgi bypass}

During Drosophila embryogenesis, dGRASP is required for unconventional secretion of $\alpha \mathrm{PS} 1$ integrin to confer adhesion to a specific plasma membrane zone during epithelial remodeling. ${ }^{22}$ The remodeling event causes a triangular void where the cells pull apart. At this stage, dGRASP and dGM130 change their localization from the Golgi to the zone of contact and nearby transitional ER, and the gap is repaired. Embryos homozygous at loss of dGRASP show a block in $\alpha$ PS1 secretion and a disorganized epithelium. Because dGRASP becomes localized to the plasma membrane, it may function there as a membrane tether that links membranes of unconventional secretory pathway to the plasma membrane. ${ }^{17}$ Because dGM130 is also present, it may act, as it does at the Golgi, to localize dGRASP. ${ }^{22}$ It has been speculated that unconventional secretion provides a means of modulating integrin adhesivity because glycosylation increases integrin adhesion to the extracellular matrix.

Additionally, GRASPs may play a role as chaperones of cargo, recruiting them to the unconventional secretory pathway. 


\section{Transmembrane cargo (Golgi bypass)}

Cytosolic cargo

(Autophagic secretion)

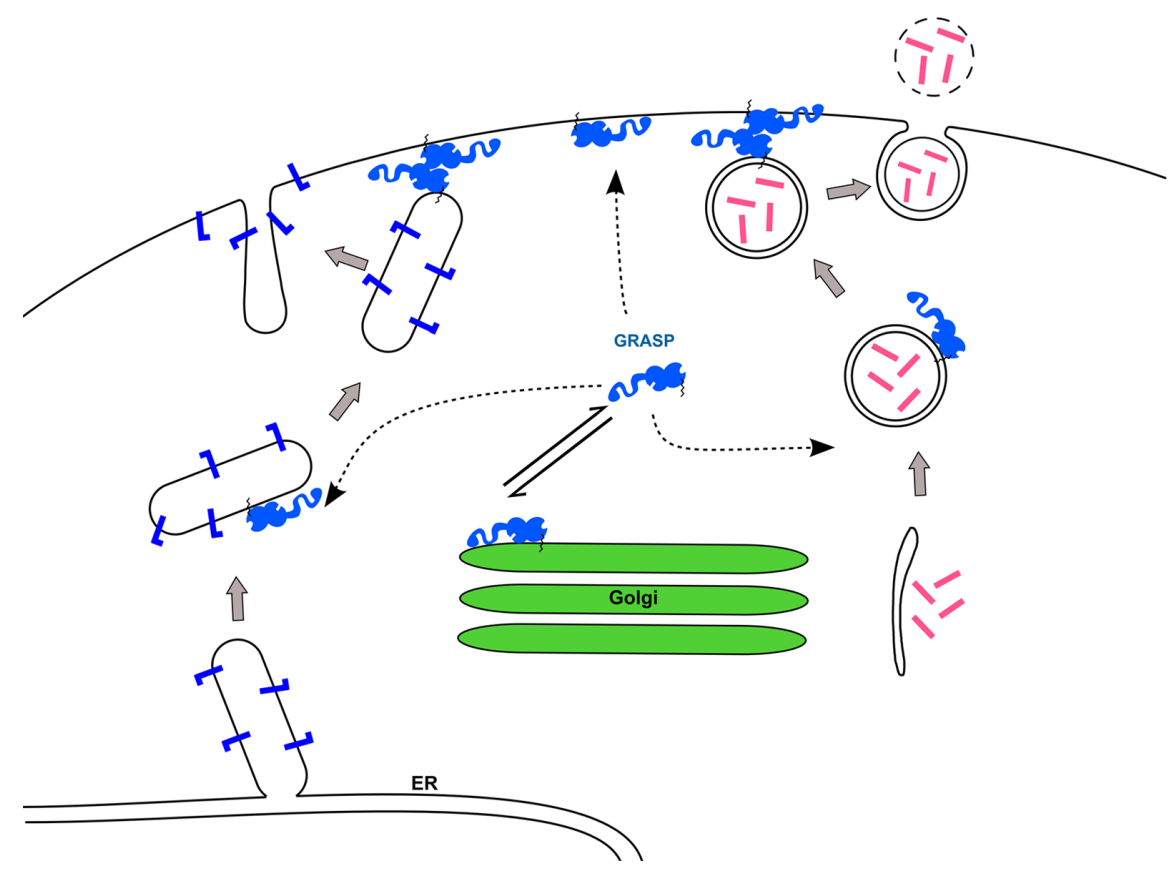

Figure 5 Models of GRASP function in unconventional secretion.

Notes: Unconventionally secreted transmembrane cargo exits the ER through a COPII independent process (left). GRASPs are recruited to the unconventional carrier as well as the plasma membrane. GRASPs then tether the membranes prior to fusion. Cytosolic cargo is first internalized into autophagic membranes (right). GRASPs are recruited to these membranes and the plasma membrane. GRASPs function as a tether prior to fusion.

Abbreviations: ER, endoplasmic reticulum; GRASP, Golgi reassembly stacking protein.

This could be similar to the binding of C-terminal valine motif cargoes in the conventional secretory pathway. ${ }^{77}$ GRASP55 depletion during interleukin-1 $\beta$ secretion decreases autophagic punctae, but not the maturation of these punctae, ${ }^{21}$ which suggests that GRASP55 plays a role early in the unconventional secretory pathway, presumably in the organization of membrane and capture of cargo.

\section{Therapeutic target?}

The tantalizing possibility of using GRASP55 to enhance unconventional secretion as a means of treating cystic fibrosis recently emerged. ${ }^{39}$ It is well known that a common form of the disease is caused by a mutation in the cystic fibrosis transmembrane conductance regulator (CFTR), which prevents its exit from the ER even though the protein retains activity. Interestingly, CFTR has a C-terminal PDZ ligand consensus sequence and undergoes unconventional secretion during the stress of an unfolded protein response. GRASP55 is required for this pathway of CFTR secretion. Furthermore, GRASP55 overexpression enhanced surface expression of the mutated CFTR, even rescuing growth defects in a mouse model. ${ }^{39}$

\section{Conclusion}

The GRASP proteins are important components in maintaining the integrity of the Golgi apparatus. In this capacity, they sustain the ribbon-like membrane network, which is important for Golgi processing and secretion kinetics, and they contribute to Golgi stacking. GRASPs are regulated during the cell cycle, in development, and in disease, which has a significant impact on the Golgi membrane network and the processes that depend on this network. Additionally, GRASPs play an important, if less understood, role in specialized secretion events. In several of these cases, there is a strong indication that PDZ interactions occur between the GRASP and the secretory cargo. However, the purpose of these interactions remains an exciting and potentially clinically significant area for future work.

\section{Disclosure}

The authors report no conflicts of interest in this work.

\section{References}

1. Bonifacino JS, Glick BS. The mechanisms of vesicle budding and fusion. Cell. 2004;116(2):153-166. 
2. Presley JF, Cole NB, Schroer TA, Hirschberg K, Zaal KJ, LippincottSchwartz J. ER-to-Golgi transport visualized in living cells. Nature. 1997;389(6646):81-85.

3. Appenzeller-Herzog C, Hauri H-P. The ER-Golgi intermediate compartment (ERGIC): in search of its identity and function. $J$ Cell Sci. 2006;119(11):2173-2183.

4. Mellman I, Simons K. The Golgi complex: in vitro veritas? Cell. 1992;68(5):829-840.

5. Lowe M. Structural organization of the Golgi apparatus. Curr Opin Cell Biol. 2011;23(1):85-93.

6. Puthenveedu MA, Bachert C, Puri S, Lanni F, Linstedt AD. GM130 and GRASP65-dependent lateral cisternal fusion allows uniform Golgienzyme distribution. Nat Cell Biol. 2006;8(3):238-248.

7. Bergmann JE, Kupfer A, Singer SJ. Membrane insertion at the leading edge of motile fibroblasts. Proc Natl Acad Sci U S A. 1983;80(5): 1367-1371.

8. Kupfer A, Louvard D, Singer SJ. Polarization of the Golgi apparatus and the microtubule-organizing center in cultured fibroblasts at the edge of an experimental wound. Proc Natl Acad Sci U S A. 1982;79(8): 2603-2607.

9. Kupfer A, Dennert G, Singer SJ. Polarization of the Golgi apparatus and the microtubule-organizing center within cloned natural killer cells bound to their targets. Proc Natl Acad Sci U S A. 1983;80(23): 7224-7228.

10. de Anda FC, Pollarolo G, Da Silva JS, Camoletto PG, Feiguin F, Dotti CG. Centrosome localization determines neuronal polarity. Nature. 2005;436(7051):704-708.

11. Feinstein TN, Linstedt AD. GRASP55 regulates Golgi ribbon formation. Mol Biol Cell. 2008;19(7):2696-2707.

12. Colanzi A, Hidalgo Carcedo C, Persico A, et al. The Golgi mitotic checkpoint is controlled by BARS-dependent fission of the Golgi ribbon into separate stacks in G2. EMBO J. 2007;26(10): 2465-2476.

13. Jesch SA, Linstedt AD. The Golgi and endoplasmic reticulum remain independent during mitosis in HeLa cells. Mol Biol Cell. 1998;9(3): 623-635.

14. Lane JD, Lucocq J, Pryde J, et al. Caspase-mediated cleavage of the stacking protein GRASP65 is required for Golgi fragmentation during apoptosis. J Cell Biol. 2002;156(3):495-509.

15. Bisel B, Wang Y, Wei J-HJH, et al. ERK regulates Golgi and centrosome orientation towards the leading edge through GRASP65. J Cell Biol. 2008;182(5):837-843

16. Nickel W. The mystery of nonclassical protein secretion. Eur J Biochem. 2003;270(10):2109-2119.

17. Giuliani F, Grieve A, Rabouille C. Unconventional secretion: a stress on GRASP. Curr Opin Cell Biol. 2011;23(4):498-504.

18. Prudovsky I, Bagala C, Tarantini F, et al. The intracellular translocation of the components of the fibroblast growth factor 1 release complex precedes their assembly prior to export. J Cell Biol. 2002;158(2): 201-208.

19. Zehe C, Engling A, Wegehingel S, Schäfer T, Nickel W. Cell-surface heparan sulfate proteoglycans are essential components of the unconventional export machinery of FGF-2. Proc Natl Acad Sci USA. 2006;103(42):15479-15484.

20. Keller M, Rüegg A, Werner S, Beer H-D. Active caspase-1 is a regulator of unconventional protein secretion. Cell. 2008;132(5): 818-831.

21. Dupont N, Jiang S, Pilli M, Ornatowski W, Bhattacharya D, Deretic V. Autophagy-based unconventional secretory pathway for extracellular delivery of IL-1 $\beta$. EMBO J. 2011;30(23):4701-4711.

22. Schotman H, Karhinen L, Rabouille C. dGRASP-mediated noncanonical integrin secretion is required for drosophila epithelial remodeling. Dev Cell. 2008;14(2):171-182.

23. Duran JM, Anjard C, Stefan C, Loomis WF, Malhotra V. Unconventional secretion of Acb1 is mediated by autophagosomes. J Cell Biol. 2010;188(4):527-536.
24. Manjithaya R, Anjard C, Loomis WFWF, Subramani S. Unconventional secretion of Pichia pastoris Acb1 is dependent on GRASP protein, peroxisomal functions, and autophagosome formation. $J$ Cell Biol. 2010;188(4):537-546.

25. Kinseth MA, Anjard C, Fuller D, Guizzunti G, Loomis WF, Malhotra V. The Golgi-associated protein GRASP is required for unconventional protein secretion during development. Cell. 2007;130(3):524-534.

26. Cabral M, Anjard C, Malhotra V, Loomis WF, Kuspa A. Unconventional secretion of AcbA in Dictyostelium discoideum through a vesicular intermediate. Eukaryot Cell. 2010;9(7):1009-1017.

27. Cooper DN. Evidence for export of a muscle lectin from cytosol to extracellular matrix and for a novel secretory mechanism. J Cell Biol. 1990;110(5):1681-1691.

28. Sato S, Burdett I, Hughes RC. Secretion of the baby hamster kidney 30-kDa galactose-binding lectin from polarized and nonpolarized cells: a pathway independent of the endoplasmic reticulum-Golgi complex. Exp Cell Res. 1993;207(1):8-18.

29. Hughes RC. Secretion of the galectin family of mammalian carbohydratebinding proteins. Biochim Biophys Acta. 1999;1473(1):172-185.

30. Mehul B, Bawumia S, Hughes RC. Cross-linking of galectin 3, a galactose-binding protein of mammalian cells, by tissue-type transglutaminase. FEBS Lett. 1995;360(2):160-164.

31. Nickel W. Unconventional secretory routes: direct protein export across the plasma membrane of mammalian cells. Traffic. 2005;6(8): 607-614.

32. Shorter J, Watson R, Giannakou ME, Clarke M, Warren G, Barr FA. GRASP55, a second mammalian GRASP protein involved in the stacking of Golgi cisternae in a cell-free system. EMBO J. 1999;18(18): 4949-4960.

33. Wang CK, Pan L, Chen J, Zhang M. Extensions of PDZ domains as important structural and functional elements. Protein Cell. 2010;1(8):737-751.

34. Oschkinat H. A new type of PDZ domain recognition. Nat Struct Biol. 1999;6(5):408-410.

35. Hillier BJ, Christopherson KS, Prehoda KE, Bredt DS, Lim WA. Unexpected modes of PDZ domain scaffolding revealed by structure of nNOS-syntrophin complex. Science. 1999;284(5415):812-815.

36. Zhang Y, Appleton BA, Wiesmann C, et al. Inhibition of Wnt signaling by disheveled PDZ peptides. Nat Chem Biol. 2009;5(4): 217-219

37. Runyon ST, Zhang Y, Appleton BA, et al. Structural and functional analysis of the PDZ domains of human HtrA1 and HtrA3. Protein Sci. 2007;16(11):2454-2471.

38. Truschel ST, Sengupta D, Foote A, Heroux A, Macbeth MR, Linstedt AD. Structure of the membrane tethering GRASP domain reveals a unique PDZ ligand interaction that mediates Golgi biogenesis. J Biol Chem. 2011;286(23):20125-20129.

39. Gee HY, Noh SH, Tang BL, Kim KH, Lee MG. Rescue of DF508-CFTR trafficking via a GRASP-dependent unconventional secretion pathway. Cell. 2011;146(16):746-760.

40. Barr FA, Nakamura N, Warren G. Mapping the interaction between GRASP65 and GM130, components of a protein complex involved in the stacking of Golgi cisternae. EMBO J. 1998;17(12): 3258-3268

41. Short B, Preisinger C, Körner R, Kopajtich R, Byron O, Barr FA. A GRASP55-rab2 effector complex linking Golgi structure to membrane traffic. J Cell Biol. 2001;155(6):877-883.

42. Barr FA, Puype M, Vandekerckhove J, Warren G. GRASP65, a protein involved in the stacking of Golgi cisternae. Cell. 1997;91(2):253-262.

43. Sengupta D, Truschel S, Bachert C, Linstedt AD. Organelle tethering by a homotypic PDZ interaction underlies formation of the Golgi membrane network. J Cell Biol. 2009;186(1):41-55.

44. Behnia R, Barr FA, Flanagan JJJ, Barlowe C, Munro S. The yeast orthologue of GRASP65 forms a complex with a coiled-coil protein that contributes to ER to Golgi traffic. J Cell Biol. 2007;176(3): 255-261. 
45. Struck NS, Herrmann S, Langer C, et al. Plasmodium falciparum possesses two GRASP proteins that are differentially targeted to the Golgi complex via a higher- and lower-eukaryote-like mechanism. $J$ Cell Sci. 2008;121(Pt 13):2123-2129.

46. Bachert C, Linstedt AD. Dual anchoring of the GRASP membrane tether promotes trans pairing. J Biol Chem. 2010;285(21):16294-16301.

47. Nourry C, Grant SGN, Borg J-P. PDZ domain proteins: plug and play! Sci STKE. 2003;2003(179):RE7.

48. Kuo A, Zhong C, Lane W, Derynck R. Transmembrane transforming growth factor- $\alpha$ tethers to the PDZ domain-containing, Golgi membraneassociated protein p59/GRASP55. EMBO J. 2000;19(23):6427-6439.

49. Greaves J, Chamberlain LH. Palmitoylation-dependent protein sorting. J Cell Biol. 2007;176(3):249-254.

50. Sengupta D, Linstedt AD. Mitotic inhibition of GRASP65 organelle tethering involves Polo-like kinase 1 (PLK1) phosphorylation proximate to an internal PDZ ligand. J Biol Chem. 2010;285(51):39994-40003.

51. Kano F. MEK and $\mathrm{Cdc} 2$ kinase are sequentially required for Golgi disassembly in MDCK cells by the mitotic xenopus extracts. $J$ Cell Biol. 2000;149(2):357-368.

52. Feinstein TN, Linstedt AD. Mitogen-activated protein kinase kinase 1-dependent Golgi unlinking occurs in G2 phase and promotes the G2/M cell cycle transition. Mol Biol Cell. February 18, 2007:594-604.

53. Jesch SA, Lewis TS, Ahn NG, Linstedt AD. Mitotic phosphorylation of Golgi reassembly stacking protein 55 by mitogen-activated protein kinase ERK2. Mol Biol Cell. 2001;12(6):1811-1817.

54. Acharya U, Jacobs R, Peters JM, Watson N, Farquhar MG, Malhotra V. The formation of Golgi stacks from vesiculated Golgi membranes requires two distinct fusion events. Cell. 1995;82(6):895-904.

55. Acharya U, Mallabiabarrena A, Acharya JK, Malhotra V. Signaling via mitogen-activated protein kinase (MEK1) is required for Golgi fragmentation during mitosis. Cell. 1998;92(2):183-192.

56. Cheng JP, Betin VM, Weir H, Shelmani GM, Moss DK, Lane JD. Caspase cleavage of the Golgi stacking factor GRASP65 is required for Fas/CD95-mediated apoptosis. Cell Death Dis. 2010;1:e82.

57. Wang Y, Satoh A, Warren G. Mapping the functional domains of the Golgi stacking factor GRASP65. J Biol Chem. 2005;280(6):4921-4928.

58. Wang Y, Seemann J, Pypaert M, Shorter J, Warren G. A direct role for GRASP65 as a mitotically regulated Golgi stacking factor. EMBO J. 2003;22(13):3279-3290

59. Yoshimura S, Yoshioka K, Barr FA, et al. Convergence of cell cycle regulation and growth factor signals on GRASP65. J Biol Chem. 2005;280(24):23048-23056.

60. Elia AEH, Cantley LC, Yaffe MB. Proteomic screen finds pSer/pThrbinding domain localizing Plk1 to mitotic substrates. Science. 2003; 299(5610):1228-1231.

61. Lowery DM, Lim D, Yaffe MB. Structure and function of Polo-like kinases. Oncogene. 2005;24(2):248-259.

62. Barr FA, Silljé HHW, Nigg EA. Polo-like kinases and the orchestration of cell division. Nat Rev Mol Cell Biol. 2004;5(6):429-440.

63. Preisinger C, Körner R, Wind M, Lehmann WD, Kopajtich R, Barr FA. Plk1 docking to GRASP65 phosphorylated by Cdk1 suggests a mechanism for Golgi checkpoint signalling. EMBO J. 2005;24(4): 753-765.

64. Bonazzi M, Spanò S, Turacchio G, et al. CtBP3/BARS drives membrane fission in dynamin-independent transport pathways. Nat Cell Biol. 2005;7(6):570-580.
65. Xiang Y, Wang Y. GRASP55 and GRASP65 play complementary and essential roles in Golgi cisternal stacking. J Cell Biol. 2010;188(2): 237-251.

66. Glick BS, Nakano A. Membrane traffic within the Golgi apparatus. Annu Rev Cell Dev Biol. 2009;25:113-132.

67. Connerly PL, Esaki M, Montegna EA, et al. Sec16 is a determinant of transitional ER organization. Curr Biol. 2005;15(16):1439-1447.

68. Levi SK, Bhattacharyya D, Strack RL, Austin JR, Glick BS. The yeast GRASP Grh1 colocalizes with COPII and is dispensable for organizing the secretory pathway. Traffic. 2010;11(9):1168-1179.

69. Kondylis V, Spoorendonk KM, Rabouille C. dGRASP localization and function in the early exocytic pathway in drosophila S2 cells. Mol Biol Cell. 2005;16(9):4061-4072.

70. Pelletier L, Stern CA, Pypaert M, et al. Golgi biogenesis in Toxoplasma gondii. Nature. 2002;418(6897):548-552.

71. Litterman N, Ikeuchi Y, Gallardo G, et al. An OBSL1-Cul7 ubiquitin ligase signaling mechanism regulates Golgi morphology and dendrite patterning. PloS Biol. 2011;9(5):e1001060.

72. Wang Y, Wei J-H, Bisel B, Tang D, Seemann J. Golgi cisternal unstacking stimulates COPI vesicle budding and protein transport. PloS One. 2008;3(2):e1647.

73. Hiyoshi M, Suzu S, Yoshidomi Y, et al. Interaction between Hck and HIV-1 Nef negatively regulates cell surface expression of M-CSF receptor. Blood. 2008;111(1):243-250.

74. Hamilton JA. Colony-stimulating factors in inflammation and autoimmunity. Nat Rev Immunol. 2008;8(7):533-544.

75. Herbein G, Varin A. The macrophage in HIV-1 infection: from activation to deactivation? Retrovirology. 2010;7(1):33.

76. Hiyoshi M, Takahashi-Makise N, Yoshidomi Y, et al. HIV-1 Nef perturbs the function, structure, and signaling of the golgi through the Src kinase Hck. J Cell Physiol. 2012;227(3):1090-1097.

77. D'Angelo G, Prencipe L, Iodice L, et al. GRASP65 and GRASP55 sequentially promote the transport of $\mathrm{C}$-terminal valine-bearing cargos to and through the Golgi complex. J Biol Chem. 2009;284(50): 34849-34860.

78. Trucco A, Polishchuk RS, Martella O, et al. Secretory traffic triggers the formation of tubular continuities across Golgi sub-compartments. Nat Cell Biol. 2004;6(11):1071-1081.

79. Guo Y, Linstedt AD. COPII-Golgi protein interactions regulate COPII coat assembly and Golgi size. J Cell Biol. 2006;174(1):53-63.

80. Barr FA, Preisinger C, Kopajtich R, Körner R. Golgi matrix proteins interact with p24 cargo receptors and aid their efficient retention in the Golgi apparatus. J Cell Biol. 2001;155(6):885-891.

81. Dominguez M, Dejgaard K, Füllekrug J, et al. gp25 L/emp24/p24 protein family members of the cis-Golgi network bind both COP I and II coatomer. J Cell Biol. 1998;140(4):751-765.

82. Roghi C, Jones L, Gratian M, English WR, Murphy G. Golgi reassembly stacking protein 55 interacts with membrane-type (MT) 1-matrix metalloprotease (MMP) and furin and plays a role in the activation of the MT1-MMP zymogen. FEBS J. 2010;277(15):3158-3175.

83. Vu TH. Matrix metalloproteinases: effectors of development and normal physiology. Gene Dev. 2000;14(17):2123-2133.
Cell Health and Cytoskeleton

\section{Publish your work in this journal}

Cell Health and Cytoskeleton is an international, peer-reviewed open access journal focusing on all aspects of cell structure and function contributing to normal physiology and cell health and exploring the pathogenesis of cell dysfunction leading to adverse conditions and disease in the organism. The journal welcomes papers covering original research,

\section{Dovepress}

basic science, reviews and evaluations, guidelines, expert opinion and commentary, case reports and extended reports. The manuscript management system is completely online and includes a very quick and fair peerreview system, which is all easy to use. Visit http://www.dovepress.com/ testimonials.php to read real quotes from published authors. 